\title{
Planetary Nebula Dust Haloes Revealed by Herschel
}

\author{
Toshiya Ueta*, Djazia Ladjal, Katrina Exter, and the Herschel Planetary Nebula \\ Survey (HerPlaNS) team ${ }^{\dagger}$ \\ University of Denver \\ E-mail: tueta@du.edu
}

\begin{abstract}
Herschel Planetary Nebula Survey (HerPlaNS) is a far-IR imaging and spectroscopic survey of planetary nebulae, performed with the Herschel Space Observatory, aiming at (1) establishing the spatially-resolved far-IR characteristics of the target nebulae and (2) understanding the energetics and shaping history of the circumstellar nebulae. Below we briefly demonstrate the breadth and depth of the HerPlaNS data set using one of the targets, NGC 6781, as an example, and explore expectations in the era of SPICA, the next-generation far-IR mission.
\end{abstract}

The Life Cycle of Dust in the Universe: Observations, Theory, and Laboratory Experiments 18-22 November, 2013

Taipei, Taiwan

\footnotetext{
* Speaker.

${ }^{\dagger}$ The founding members of the HerPlaNS team are; T. Ueta (DU), D. Ladjal (DU), K. M. Exter (KUL), M. Otsuka (ASIAA), R. Szczerba, N. Siódmiak (NCAC), I. Aleman, A. G. G. M. Tielens (Leiden U.) J. H. Kastner, J. Nordhaus (RIT), R. Montez (Vanderbilt), I. McDonald, K. Hebden, A. Zijlstra (U. of Manchester), M. Wittkowski (ESO), S. Ramstedt (Uppsala U.), O. De Marco (Macquarie U.), E. Villaver (UAM), B. Balick (UW), E. Behar (Technion), E. G. Blackman (U. of Rochester, Y.-H. Chu (UIUC), J. L. Hora (CfA/Harvard), H. Izumiura (OAO/NAOJ), J. A. Lopez (UNAM), K. Murakawa (Leeds), R. Nordon (MPE), C. Sandin (AIP), R. Sahai (JPL), P. A. M. van Hoof (KSB/ORB), W. Vlemmings (Chalmers), and I. Yamamura (ISAS/JAXA).
} 


\section{Herschel Planetary Nebula Survey (HerPlaNS)}

The Herschel Planetary Nebula Survey (HerPlaNS; [1]) is an open-time program of the Herschel Space Observatory [2]. Our chief objective is to examine the spatially-resolved far-IR characteristics of planetary nebulae (PNs; Table 1) as energetic systems of gas and dust. Far-IR observations allow simultaneous probing of the dust component via thermal continuum emission and the gas component via far-IR fine-structure and molecular line emission without much extinction. The spatially-resolved energetics as a function of location in these nebulae will provide more insights about the evolution of the central star and circumstellar shells. In this contribution, we present a brief overview of the survey and its data products, summarizing the potential of the data set using NGC 6781 as an example ( $\$ 2)$, and hint at expectations for the era of SPICA (\$3). A complete account of the present summary is found elsewhere [1].

\section{HerPlaNS Data Demonstration with NGC 6781}

NGC 6781 is a PN near the end of the interior nuclear burning phase, whose central star (CSPN) is of $110 \mathrm{kK}$ [3] and $385 \mathrm{~L}_{\odot}$ at $950 \pm 143 \mathrm{pc}$ away [4]. Comparison of these parameters with evolutionary tracks of [5] suggests that the initial and present masses of the central star are 1.5 and $0.6 \mathrm{M}_{\odot}$, respectively, and the age of the PN since the AGB turn-off is $3 \times 10^{4} \mathrm{yr}$.

The optical nebula of NGC 6781 shows a signature "ring" of 50" radius within a diffuse, lowemission nebula extending $190^{\prime \prime} \times 160^{\prime \prime}[6,7]$. Morpho-kinematic observations in molecular lines $[8,9]$ indicated that the nebula is a nearly pole-on cylinder with an equatorial enhancement (i.e., barrel) with a dynamical age of $4 \times 10^{4} \mathrm{yr}$ at the adopted distance of $950 \mathrm{pc}$. Hence, the spatiallyresolved data of NGC 6781 will reveal variations nebular quantities in the equatorial plane as a function of radius, taking into account the height of the bipolar structure along the direction of the line of sight.

Table 1: List of HerPlaNS Target PNs

\begin{tabular}{lcccccccc}
\hline \hline Name & PN G & Morph $^{\mathrm{a}}$ & $\begin{array}{c}\mathrm{D} \\
\mathrm{kpc})\end{array}$ & $\begin{array}{c}\mathrm{R} \\
(\mathrm{pc})\end{array}$ & $\begin{array}{c}\text { Age } \\
\left(10^{3} \mathrm{yr}\right)\end{array}$ & $\begin{array}{c}\mathrm{T}_{*} \\
\left(10^{3} \mathrm{~K}\right)\end{array}$ & $\mathrm{H}_{2}$ & X-Rays ${ }^{\mathrm{b}}$ \\
\hline Mz 3 $^{d}$ & $331.7-01.0$ & Bps & $1-3$ & $0.1-0.2$ & $0.6-2$ & $32:$ & $\mathrm{Y}$ & $\mathrm{D}, \mathrm{P}$ \\
NGC 2392 & $197.8+17.3$ & Rsai & 1.3 & 0.14 & 3 & 47 & $\mathrm{~N}$ & $\mathrm{D}, \mathrm{P}$ \\
NGC 40 & $120.0+09.8$ & Bbsh & 1.0 & 0.11 & 4 & 48 & $\mathrm{~N}$ & $\mathrm{D}$ \\
NGC 6543 & $096.4+29.9$ & Mcspa & 1.5 & 0.09 & 5 & 48 & $\mathrm{~N}$ & $\mathrm{D}, \mathrm{P}$ \\
NGC 6826 & $083.5+12.7$ & Ecsah & 1.3 & 0.08 & 5 & 50 & $\mathrm{~N}$ & $\mathrm{D}, \mathrm{P}$ \\
NGC 7026 & $089.0+00.3$ & Bs & 1.7 & 0.16 & $<1$ & 80 & $\mathrm{~N}$ & $\mathrm{D}$ \\
NGC 7009 & $037.7-34.5$ & Lbspa & 1.5 & 0.09 & 3 & 87 & $\mathrm{~N}$ & $\mathrm{D}, \mathrm{P}$ \\
NGC 3242 & $261.0+32.0$ & Ecspaih & 1.0 & 0.10 & 4 & 89 & $\mathrm{~N}$ & $\mathrm{D}$ \\
NGC 6781 & $041.8-02.9$ & Bth & 1.0 & 0.32 & 26 & 112 & $\mathrm{Y}$ & $\mathrm{N}$ \\
NGC 6720 & $063.1+13.9$ & Ecsh & 0.7 & 0.13 & 6 & 148 & $\mathrm{Y}$ & $\mathrm{N}$ \\
NGC 6445 & $008.0+03.9$ & Mpi & 1.4 & 0.14 & 3 & 170 & $\mathrm{Y}$ & $\mathrm{P}$ \\
\hline
\end{tabular}

(a) According to the morphological classification by [10]. (b) The HerPlaNS sample is a subset of the Chandra X-ray Planetary Nebula Survey [11]: D - diffuse, P - point-source X-ray detection. (c) Not a ChanPlaNS target PN. The PSF of XMM-Newton does not allow clear determination of the presence of a point source. (d) Not a ChanPlaNS target PN. 

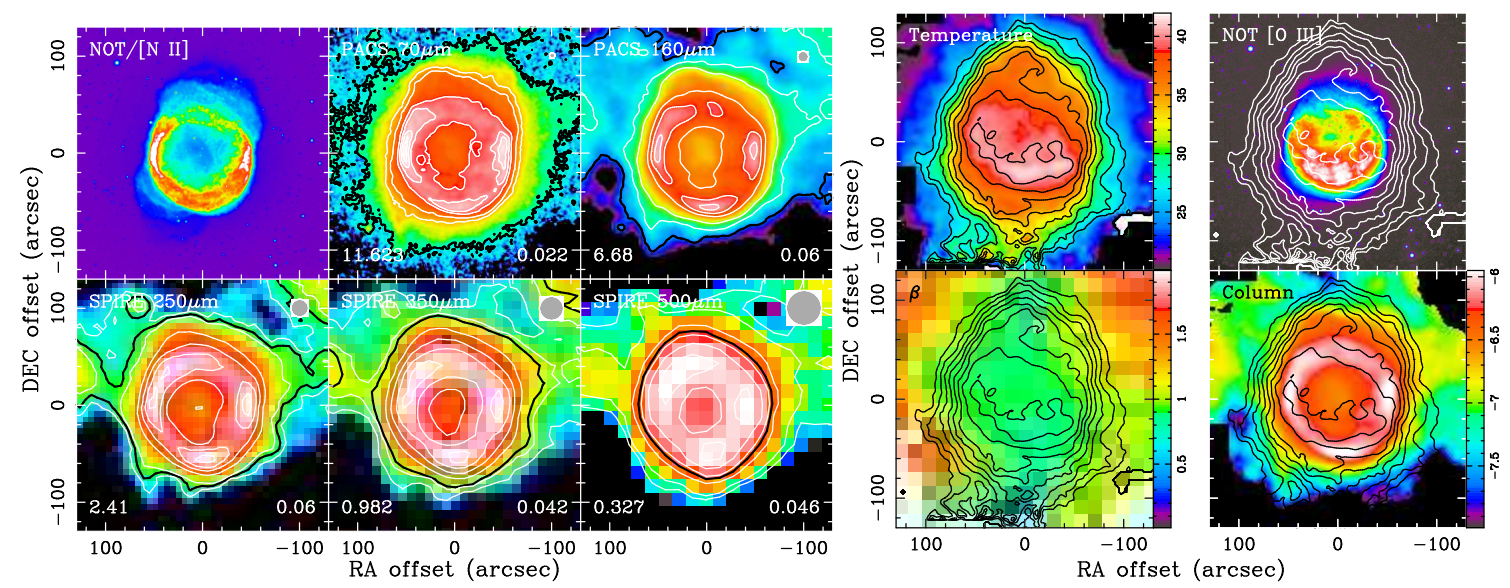

Figure 1: [Left] HerPlaNS broadband far-IR images of NGC 6781 with an [N II] image taken at the Nordic Optical Telescope (NOT) [7]. The far-IR peak surface brightness and 1- $\sigma$ sky noise in mJy $\operatorname{arcsec}^{-2}$ are indicated at the bottom-left and right corners, respectively. At the top-right corner, the beam is shown: 5.61, $11.39,18.2,24.9$, and $36.3^{\prime \prime}$, respectively. Contours are $90,70,50,30,10$, and $5 \%$ of the peak, and the black contour indicates 3- $\sigma$ threshold. The pixel scales are 1, 1, 2, 6, 9, and $14 \operatorname{arcsec}_{\text {pix }}{ }^{-1}$, respectively, from top left to bottom right. [Right] (Top Left) The dust temperature ( $\left.T_{\text {dust }}\right)$ map of NGC 6781 at the $11.4^{\prime \prime}$ resolution derived by a power-law dust emissivity fit. The peak is $41.3 \mathrm{~K}$, with contours at a $2 \mathrm{~K}$ interval from 40 to $26 \mathrm{~K}$. These $T_{\text {dust }}$ contours are always overlaid in other frames. (Top Right) the NOT [O III] $\lambda 5007$ map showing the high-ionization regions. (Bottom Left) The power-law index ( $\beta$ ) map at the 36.3" resolution. (Bottom Right) The dust column mass density $\left(\mathrm{M}_{\odot} \mathrm{pix}^{-1}\right)$ map at the $11.4^{\prime \prime}$ resolution.

\subsection{Broadband Imaging: Diagnostics via Dust Continuum}

HerPlaNS broadband imaging consists of PACS imaging of a $600^{\prime \prime} \times 600^{\prime \prime}$ field at 70 and $160 \mu \mathrm{m}$ and SPIRE imaging of a $240^{\prime \prime} \times 480^{\prime \prime}$ field at 250, 350, and $500 \mu \mathrm{m}$. Far-IR images of NGC 6781 (Figure 1, left) reveal the signature ring of the near pole-on cylindrical barrel. The total fluxes are measured by aperture photometry with the $3-\sigma_{\text {sky }}$ threshold (Table 2$)$. The surface brightness distribution at $70 \mu \mathrm{m}$ is co-spatial with low-ionization optical line emission. The emission peaks at the eastern and western rims represent the pivot points of the inclined barrel. The extent of the far-IR emission is about the same as the diffuse [N II] emission of a deep exposure image (about $100^{\prime \prime}$ radius at $5-\sigma ;[6]$ ).

Table 2: Far-IR Image Characteristics and Photometry of NGC 6781

\begin{tabular}{lccccc}
\hline Band & $\begin{array}{c}\lambda \\
(\mu \mathrm{m})\end{array}$ & $\begin{array}{c}\Delta \lambda \\
(\mu \mathrm{m})\end{array}$ & $\begin{array}{c}I_{\text {peak }} \\
\left(\mathrm{mJy} \mathrm{arcsec}^{-2}\right)\end{array}$ & $\begin{array}{c}\sigma_{\text {sky }} \\
\left(\mathrm{mJy} \mathrm{arcsec}^{-2}\right)\end{array}$ & $\begin{array}{c}F_{V} \\
(\mathrm{Jy})\end{array}$ \\
\hline PACS Blue & 70 & 25 & 11.623 & 0.022 & $65.42 \pm 3.28$ \\
PACS Red & 160 & 85 & 6.68 & 0.06 & $64.88 \pm 3.28$ \\
SPIRE PSW & 250 & 76 & 2.41 & 0.06 & $30.04 \pm 4.60$ \\
SPIRE PMW & 350 & 103 & 0.982 & 0.042 & $14.56 \pm 2.25$ \\
SPIRE PLW & 500 & 200 & 0.327 & 0.046 & $6.41 \pm 1.02$ \\
\hline
\end{tabular}


By fitting these emission maps with the power-law dust emissivity $\left(S_{v} \propto \lambda^{-\beta} B_{v}\left(T_{\text {dust }}\right)\right.$, where $\beta$ is the dust emissivity index and $T_{\text {dust }}$ is the temperature), we successively derive the $\beta, T_{\text {dust }}$, optical depth, and dust column mass density maps (Figure 1, right). The high $T_{\text {dust }}$ region ( $\gtrsim 40 \mathrm{~K}$ ) is co-spatial with the [O III] $\lambda 5007$ line emission, delineating the highly-ionized inner barrel cavity. The $\beta$ map is almost entirely unity, suggesting a carbon-based nature of dust [12], consistent with the previous assessments [7, 15].

Because far-IR thermal dust continuum is optically thin $\left(\tau_{160 \mu \mathrm{m}}=10^{-5}\right.$ to $10^{-6}$ on the "ring"), the dust column mass density map probes the whole nebula depth along the line of sight, corroborating the pole-on barrel structure previously only inferred and modeled with optical data. By integrating over the entire nebula, the total amount of far-IR emitting dust is determined to be $M_{\text {dust }}=3.8 \times 10^{-3} \mathrm{M}_{\odot}$ and roughly $50 \%$ of which appears contained in the cylindrical barrel.

\subsection{Spatio-Spectroscopy: Diagnostics via Gas Emission}

HerPlaNS spectroscopy includes PACS integral-field over 51-220 $\mu \mathrm{m}$, resulting in 25 spectra over a $47^{\prime \prime} \times 47^{\prime \prime}$ field and SPIRE Fourier-transform spectroscopy resulting in 35 spectra in the SSW band (194-313 $\mu \mathrm{m}$ ) and 19 spectra in the SLW band (303-672 $\mu \mathrm{m}$ ) over a field of a $\sim 120^{\prime \prime}$ radius. Figure 2 shows spectra for the whole 51-672 $\mu \mathrm{m}$ at two spatial pointings (at the center and eastern rim), at which continuum is detected at about $\lesssim 10 \mathrm{mJy} \operatorname{arcsec}^{-2}$ in the PACS range and less than a few mJy $\operatorname{arcsec}^{-2}$ in the SPIRE range. Thermal dust continuum emission in the PACS range is generally stronger at the eastern rim, but is about the same at both pointings in the SPIRE range. This indicates that a cold dust envelope surrounds the central highly-ionized cavity.

Besides continuum, a plethora of lines are detected. The relative line strengths of the two pointings suggest that the central cavity is more strongly ionized. While rich in lines, we determine that line contamination in broadband images is at most $20 \%$ based on these spectra. Moreover, PACS/SPIRE spectra taken by arrays of spaxels/bolometers can be rendered into spectral line maps by integrating the data cube over specific emission lines (Figure 3). In the case of NGC 6781, we derived the electron temperature $\left(T_{\mathrm{e}}\right)$ and electron density $\left(n_{\mathrm{e}}\right)$ maps using far-IR line ratio maps (e.g., [O III] $52 \mu \mathrm{m} / 88 \mu \mathrm{m}$ and [N II] $122 \mu \mathrm{m} / 205 \mu \mathrm{m}$ ) augmented with other optical-to-far-IR line ratio maps (e.g., [O III] $\lambda 5007 / 88 \mu \mathrm{m}$ and [N II] $\lambda 6583 / 122 \mu \mathrm{m}$ ) by solving the equation of statistical equilibrium [13-15]. Moreover, these $\left(T_{\mathrm{e}}, n_{\mathrm{e}}\right)$ maps can be translated into ionic/elemental abundance maps [16]. Here we show 1-D profiles along the cylindrical radius (Figure 3). The $T_{\mathrm{e}}$ profiles reveals constant $T_{\mathrm{e}}(\sim 9,000 \mathrm{~K})$ in the highly-ionized barrel cavity, surrounded by the $T_{\mathrm{e}}$

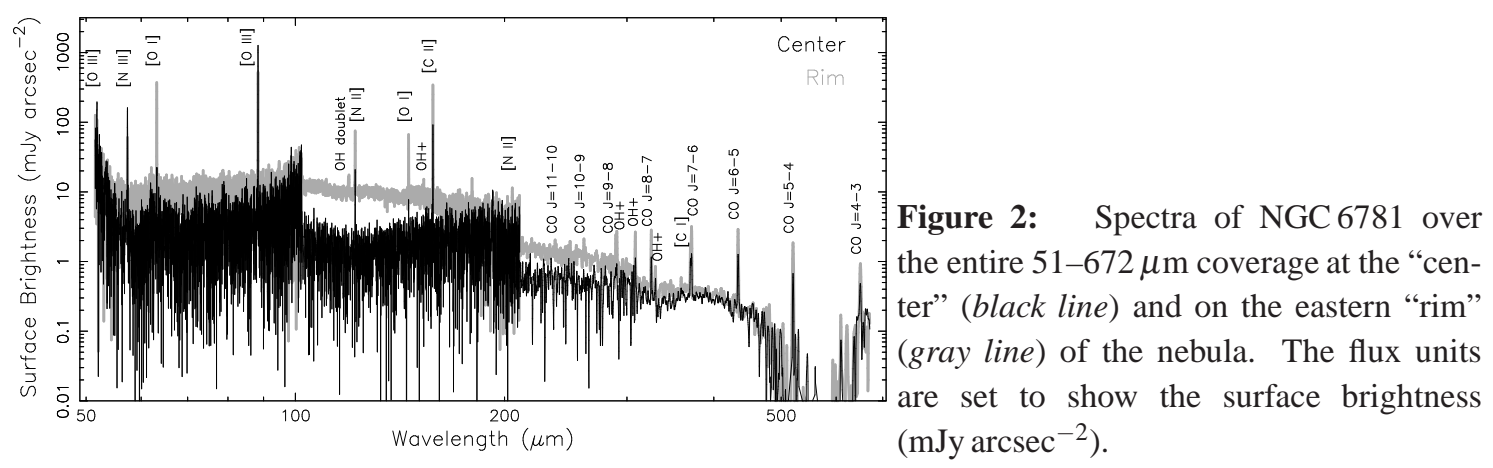



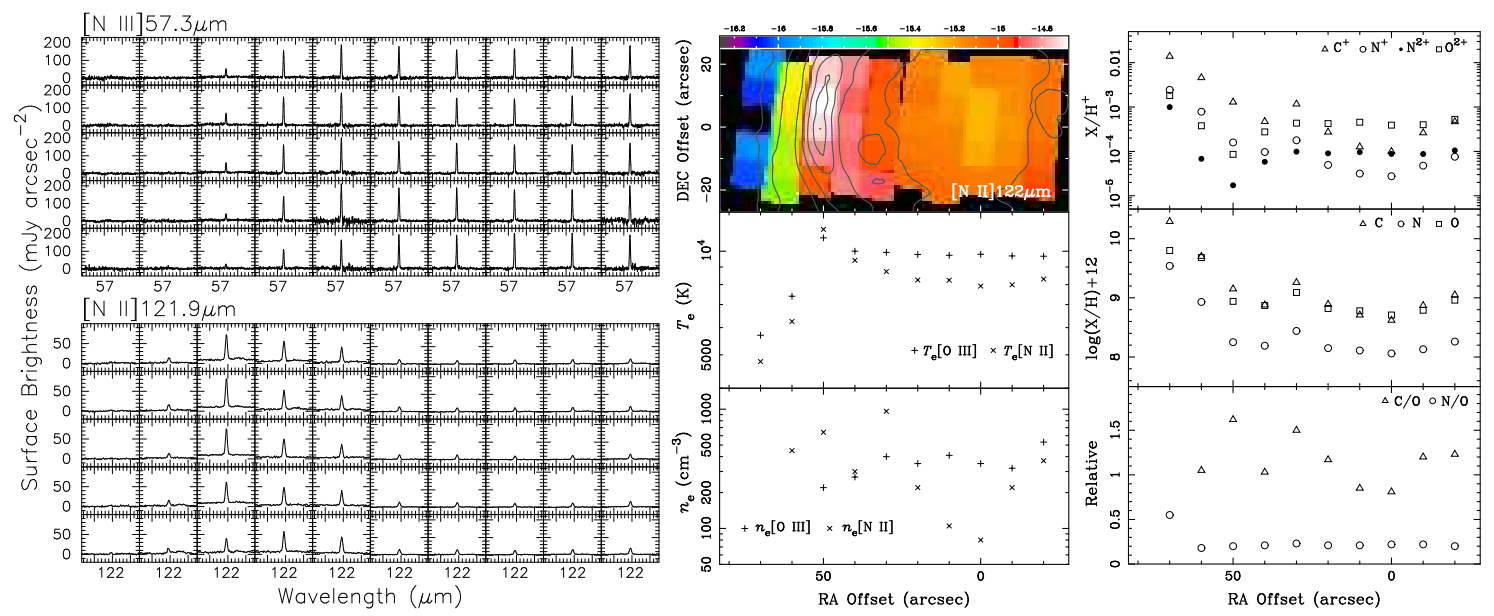

Figure 3: (Left) Spatial variation of [N III] $57 \mu \mathrm{m}$ (top) and [N II] $122 \mu \mathrm{m}$ (bottom) line emission in individual spectra. (Middle) The [N II] $122 \mu \mathrm{m}$ line intensity map (top in hue). The $\log T_{\mathrm{e}}$ profiles (middle). The $\log n_{\mathrm{e}}$ profiles (bottom). (Right) The relative ionic abundance profiles (top). The relative elemental abundance profiles (where $\log N_{\mathrm{H}}=12 ;$ middle). The $\mathrm{C} / \mathrm{O}$ and N/O profiles (bottom). The legend is given in each frame - Crosses: $T_{\mathrm{e}}, n_{\mathrm{e}}$ based on [O III], Pluses: $T_{\mathrm{e}}, n_{\mathrm{e}}$ based on [N II], Triangles: $\mathrm{C}^{+}, \log (\mathrm{C} / \mathrm{H})+12$, and C/O, Circles: $\mathrm{N}^{+}$(open), $\mathrm{N}^{2+}$ (filled), $\log (\mathrm{N} / \mathrm{H})+12$, and $\mathrm{N} / \mathrm{O}$, Squares: $\mathrm{O}^{2+}$ and $\log (\mathrm{O} / \mathrm{H})+12$.

maximum $(\sim 10,500 \mathrm{~K})$ beyond which $T_{\mathrm{e}}$ tapers off. The $n_{\mathrm{e}}$ profiles show a uniform distribution of a high-ionization gas $n_{\mathrm{e}}$ [O III] (pluses; $\sim 400 \mathrm{~cm}^{-3}$ ) and a radially increasing distribution of a low-ionization gas $n_{\mathrm{e}}\left[\mathrm{N}\right.$ II] (crosses; $100-600 \mathrm{~cm}^{-3}$ ).

Moreover, the ionic abundance profiles reveal the physical stratification: the highly ionized cavity $\left(\lesssim 30^{\prime \prime}\right)$ in which $\mathrm{O}^{+}(>35.1 \mathrm{eV})$ and $\mathrm{N}^{+}(>29.6 \mathrm{eV})$ are ionized, the inner barrel wall $(\sim$ $\left.50^{\prime \prime}\right)$ in which $\mathrm{C}^{+}(>24.4 \mathrm{eV})$ and $\mathrm{N}^{0}(>14.5 \mathrm{eV})$ are ionized, and beyond the barrel wall $\left(\gtrsim 50^{\prime \prime}\right)$. Similarly, the $\mathrm{CNO}$ elemental abundance profiles are derived. The marginal $\mathrm{N}$ abundance (not Type $\mathrm{I}$, as $\mathrm{N} / \mathrm{O} \lesssim 0.5$; [17]) suggests that the CSPN is most likely less than $2 \mathrm{M}_{\odot}$ initial mass.

\section{Future Prospects}

By combining the above analyses of broadband and line maps, we can empirically determine the gas and dust column maps independently (Figure 4). For NGC 6781, the derived dust-to-gas mass ratio distribution is in general consistent with a typical ballpark figure of roughly 400 for carbon-rich AGB winds [18]. However, we note that the ratio varies radially from over 550 to 100 over the cylindrical barrel wall. Hence, we may need to reconsider the presently rampant "onevalue-fits-all" approach of extrapolating the unknown amount of gas or dust component by scaling the empirically determined amount of the other component with a single gas-to-dust mass ratio. With the rich HerPlaNS data set, we will decipher the spatially-resolved physical stratifications in these dusty gaseous systems. These insights will enhance the understanding of not only the PN physics itself but also the general far-IR line diagnostics used for systems that are not necessarily spatially resolved (e.g., luminous infrared galaxies; for which many excellent reports can be found in the present proceedings issue). 


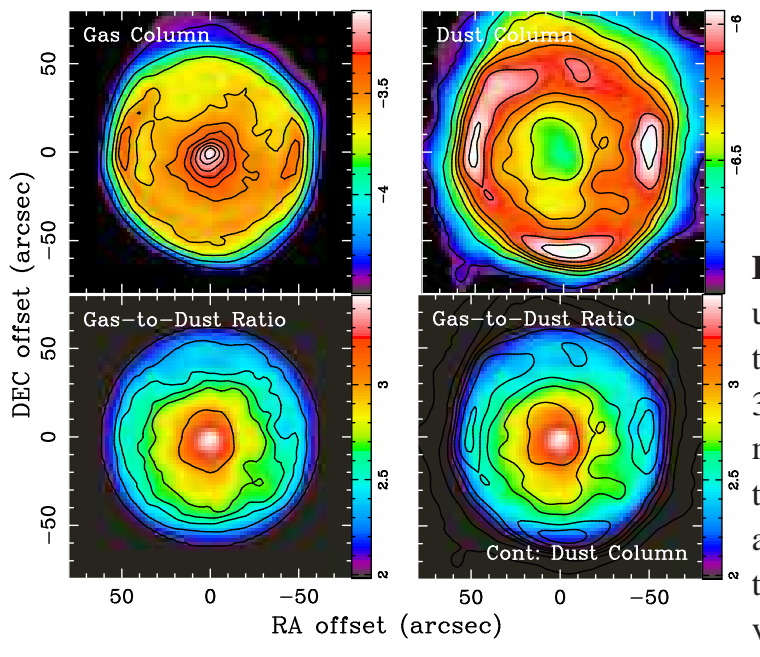

\section{Acknowledgments}

Major support for this work was provided by NASA through an award issued by JPL/Caltech, by the Japan Society for the Promotion of Science through an FY2013 long-term invitation fellowship program, and by the Belgian Federal Science Policy Office via the ESA PRODEX Programme.

\section{References}

[1] T. Ueta, et al., $A \& A$, in press

[2] G. L. Pilbratt, et al., $A \& A$, 518, L1 (2010)

[3] D. J. Frew, Ph.D. Thesis, Macquarie University (2008)

[4] H. E. Schwarz \& Monteiro H. ApJ, 648, 430 (2006)

[5] E. Vassiliadis \& P. R. Wood, ApJS, 92, 125 (1994)

[6] F. Mavromatakis, J. Papamastorakis, \& E. V. Paleologou, A\&A, 374, 280 (2001)

[7] J. P. Phillips, G. Ramos-Larios, \& M. A. Guerrero, MNRAS, 415, 513 (2011)

[8] R. Bachiller, P. J. Huggins, P. Cox, \& T. Forveille, A\&A, 267, 177 (1993)

[9] D. Hiriart, $A \& A, \mathbf{1 8 7}, 181$ (2005)

[10] R. Sahai, M. R. Morris, \& G. G. Villar, AJ, 141, 134 (2011)

[11] J. H. Kastner, et al. AJ, 144, 58 (2012)

[12] K. Volk \& S. Kwok, ApJ, 331, 435 (1988)

[13] X.-W. Liu, et al. MNRAS, 323, 343 (2001)

[14] Y. Liu, X.-W. Liu, S.-G. Luo, \& M. J. Barlow, MNRAS, 353, 1231 (2004)

[15] Y. Liu, X.-W. Liu, M. J. Barlow, \& S.-G. Luo, MNRAS, 353, 1251 (2004)

[16] C. Vamvatira-Nakou, et al., $A \& A, \mathbf{5 5 7}$, A20 (2013)

[17] M. Peimbert \& S. Torres-Peimbert, in Planetary Nebulae, edited by D. R. Flower, 233 (1983)

[18] G. R. Knapp, ApJ, 293, 273 (1985) 